\title{
DFT Studies on Structural Stability and Magnetic Properties of $\mathrm{XTiSb}(\mathrm{X}=\mathrm{Fe}, \mathrm{Co})$
}

\author{
R. Murugeswari ${ }^{1}$, A.Milton Franklin Benial ${ }^{1}$, R. Rajeswarapalanichamy ${ }^{1 *}$ \\ ${ }^{I}$ Department of Physics, N.M.S.S.V.N college, Madurai, Tamilnadu-625019, India
}

\begin{abstract}
The structural stability of titanium based magnetic materials XTiSb $(X=F e, C o)$ is investigated by calculating the total energies for both magnetic and nonmagnetic phases by the first principles calculations based on density functional theory using Vienna ab-initio simulation package (VASP). The ground state parameters such as lattice constant, cell volume and bulk modulus are calculated. The calculated lattice parameters are in agreement with earlier reported values. The mechanical parameters like elastic constants, bulk modulus, Youngs modulus, Poisson's ratio and B/G ratio are estimated. The calculated magnetic moments of FeTiSb and CoTiSb are $0.906 \mu_{B}$ and $0.001 \mu_{B}$, which indicate that FeTiSb is stable in magnetic phase and CoTiSb is in nonmagnetic phase. The density of states reveals that FeTiSb is a half metallic with an energy gap of $0.576 \mathrm{eV}$ and CoTiSb is a semiconductor with an energy gap of $1.013 \mathrm{eV}$ at ambient condition.
\end{abstract}

Keywords: Ab-initio calculation, Electronic structure, Magnetic property, Mechanical properties.

\section{Introduction}

Titanium and its alloys are attractive metallic materials which are widely used as implants for dental, restorations and orthodontic wires as well as orthopaedic due to their non-corrosive, low density and biocompatibility [1]. Titanium alloys have low modulus, good fatigue strength, formability, machinability, low thermal conductivity, high strength to weight ratio, than in many other structural materials, which have made them desirable for industry, medicine, dentistry and aeronautical applications [2]. Titanium based ternary intermetallic half heusler alloys XYZ crystallized in the cubic $\left(\mathrm{CI}_{\mathrm{b}}\right)$ structure have attracted more attention because they posses wide variety of interesting physical properties such as very high seeback coefficient, electrical resistivity and thermal conductivity. In the present work, the structural, mechanical, electronic and magnetic properties of Titanium based Half Heusler magnetic materials FeTiSb and CoTiSb for possible nonmagnetic and magnetic phases are reported.

\section{Computational Details}

In this work, all the calculations are performed using the Vienna Ab-initio Simulation Package (VASP) code within the density functional theory frame work, where the PBE form of GGA [3,4] was employed to describe the electron exchange and correlation. The calculations are carried out with an energy cut-off $350 \mathrm{eV}$ for both the alloys and the Brillouin zone integration performed with a mesh of 4X4X4 generated by Monkhorst-pack method for both FeTiSb and CoTiSb alloys. The valance electronic configuration for FeTiSb and CoTiSb are as Ti: $3 d^{2} 4 s^{2}$, Fe: $3 d^{6} 4 s^{2}$, Co: $3 d^{7} 4 s^{2}$ and Sb: $5 s^{2} 5 p^{3}$ respectively.

\subsection{Ground State Properties}

\section{Results And Discussion}

The lattice constants are optimized and the total energy is calculated for FeTiSb and CoTiSb alloys in nonmagnetic and magnetic phases. The calculated ground state properties such as equilibrium volume $\left(\mathrm{V}_{0}\right)$, lattice constant (a), valance electron density $(\rho)$, bulk modulus $\left(\mathrm{B}_{0}\right)$, bulk modulus derivative (B') and Fermi energy $\left(\mathrm{E}_{\mathrm{F}}\right)$ for FeTiSb and CoTiSb for are given in Table 1 along with available results $[5,6]$. 
Table1. The equilibrium volume $\mathrm{V}_{0}\left(\AA^{3}\right)$, optimized lattice constant a $(\AA)$, Valence electron density $\rho$ (electrons/ $\left.\AA^{3}\right)$, bulk modulus $\mathrm{B}(\mathrm{GPa})$ and its derivative(B') and Fermi energy $\mathrm{E}_{\mathrm{F}}(\mathrm{eV})$.

\begin{tabular}{lllll}
\hline & \multicolumn{3}{c}{ FeTiSb } & CoTiSb \\
\cline { 2 - 5 } & NM & FM & NM & FM \\
\hline $\mathrm{V}_{0}$ & 51.890 & 52.980 & 50.980 & 50.980 \\
& & & $51.250^{\mathrm{a}}$ & \\
$\mathrm{a}$ & 5.921 & 5.962 & 5.886 & 5.886 \\
& $5.893^{\mathrm{b}}$ & $5.897^{\mathrm{b}}$ & $5.838^{\mathrm{b}}$ & $5.826^{\mathrm{b}}$ \\
& & $5.957^{\mathrm{c}}$ & $5.884^{\mathrm{c}}$ & \\
$\rho$ & 0.327 & 0.327 & 0.353 & 0.353 \\
$\mathrm{~B}_{0}$ & 124.705 & 101.859 & 122.517 & 122.518 \\
$\mathrm{~B}^{\prime}$ & 5.829 & 5.834 & 5.848 & 5.836 \\
$\mathrm{E}_{\mathrm{F}}$ & 6.479 & 6.480 & 6.319 & 6.323 \\
\hline
\end{tabular}

${ }^{\mathrm{a}} \operatorname{Ref}[5],{ }^{\mathrm{b}} \operatorname{Ref}[6],{ }^{\mathrm{c}} \operatorname{Ref}[7]$

\subsection{Structural Phase transition}

The structural stability of FeTiSb and CoTiSb alloys of both nonmagnetic and magnetic phases is analyzed under normal and high pressure. The lattice constants are optimized and the total energy is calculated for both FeTiSb and CoTiSb alloys for nonmagnetic and magnetic phases as a function of cell volume and their values are given in Table 2. FeTiSb adopt the magnetic phase of cubic $\left(\mathrm{CI}_{b}\right)$ as the most stable structure whereas CoTiSb possess the nonmagnetic cubic $\left(\mathrm{CI}_{\mathrm{b}}\right)$ structure which both have the symmetry of the F-43m $(216)$ space group.

Table 2. Listed the nonmagnetic and magnetic energies (eV) of FeTiSb and CoTiSb alloys with function of cell volume $\left(\AA^{3}\right)$.

\begin{tabular}{|c|c|c|c|c|c|c|}
\hline \multicolumn{4}{|c|}{ FeTiSb } & \multicolumn{3}{|c|}{ CoTiSb } \\
\hline Cell volume & NM & Cell volume & FM & Cell volume & NM & FM \\
\hline 51.89 & -21.222856 & 52.98 & -21.299408 & 50.98 & -20.974511 & -20.970342 \\
\hline 46.69 & -20.943192 & 47.68 & -21.060186 & 45.86 & -20.688471 & -20.685324 \\
\hline 41.50 & -19.848984 & 42.37 & -20.112599 & 40.78 & -19.584768 & -19.581713 \\
\hline 36.32 & -17.326899 & 37.07 & -17.816728 & 35.68 & -17.018782 & -17.015265 \\
\hline 31.12 & -12.204462 & 31.79 & -13.071123 & 30.58 & -11.792953 & -11.791505 \\
\hline 25.94 & -2.275234 & 26.49 & -3.668979 & 25.48 & -1.532873 & -1.530629 \\
\hline
\end{tabular}

\subsection{Mechanical Properties}

The elastic constants $\mathrm{C}_{\mathrm{ij}}$, bulk modulus $\mathrm{B}_{0}$, bulk modulus derivative B', Young's modulus E, Energy gap $\mathrm{E}_{\mathrm{g}}$, Magnetic moment $\mathrm{M}, \mathrm{B} / \mathrm{G}$ ratio and Poisson's ratio ( $v$ ) for the stable phase of FeTiSb and CoTiSb alloys are listed in Table 3. The calculated Young's modulus and bulk modulus of both the alloys indicates that $\mathrm{CoTiSb}$ is stiffer and stronger capacity to resist deformation than FeTiSb. The larger Poisson's ratio value clearly indicates that ionic contribution is dominant in XTiSB (X=Fe, Co) alloys. The calculated (B/G) values of XTiSb $(\mathrm{X}=\mathrm{Fe}, \mathrm{Co})$ alloys show that both FeTiSb and CoTiSb alloys posses the brittle nature at zero pressure.

\subsection{Electronic properties}

The spin polarized and total density of states (DOS) of FeTiSb and CoTiSb is given in Fig.1 (a) \& (b) and Fig. 2 (a) \& (b) respectively. The peaks observed at lower energy region is due to the Fe-3d, Co- $3 \mathrm{~d}$ and $\mathrm{Sb}$ 5 s states, and the peaks at higher energy region are due to the $3 \mathrm{~d}$ states of Ti. In Fig 1(a) shows that there is no symmetry between spin up $(\uparrow)$ and spin down $(\downarrow)$ of the spin polarized DOS of FeTiSb, which clearly explains that FeTiSb exhibits the half metallic nature with an energy gap of $0.576 \mathrm{eV}$. In Fig 2(a) spin up ( $\uparrow$ ) and spin down $(\downarrow)$ of the CoTiSb posses the symmetry nature. [7]. In CoTiSb alloy, Sb atom has five valence electrons and $\mathrm{Co}$ and $\mathrm{Ti}$ totally have 13 valence electrons. Among these 13 transition metal electrons only three are catched by the $\mathrm{Sb}$ atom and the remaining 10 electrons occupy the bonding $\mathrm{d}$ states. So, CoTiSb is a semiconductor with an energy gap of $1.013 \mathrm{eV}$. 
Table 3. Listed the elastic constants $\mathrm{C}_{\mathrm{ij}}(\mathrm{GPa})$, bulk modulus $\mathrm{B}_{0}(\mathrm{GPa})$, bulk modulus derivative $\mathrm{B}$ '(GPa),

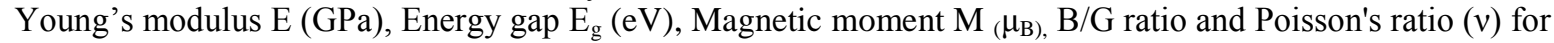
the stable phase of FeTiSb and CoTiSb alloys.

\begin{tabular}{|c|c|c|c|c|c|c|c|c|c|c|}
\hline & $\mathrm{C}_{11}$ & $\mathrm{C}_{12}$ & $\mathrm{C}_{44}$ & $\mathrm{~B}_{0}$ & $\mathrm{~B}_{0}{ }^{\prime}$ & E & $E_{g}$ & M & $\mathrm{B} / \mathrm{G}$ & $v$ \\
\hline $\begin{array}{l}\text { FeTisb } \\
(\mathrm{FM})\end{array}$ & 257.317 & 46.739 & 71.792 & $\begin{array}{l}116.931 \\
132.53^{\mathrm{a}}\end{array}$ & $\begin{array}{l}5.834 \\
4.95^{\mathrm{a}}\end{array}$ & 205.6345 & 0.576 & $\begin{array}{l}0.906 \\
0.82^{\mathrm{a}}\end{array}$ & 1.372 & 0.206 \\
\hline $\begin{array}{l}\text { CoTiSb } \\
\text { (NM) }\end{array}$ & $\begin{array}{l}269.856 \\
259^{\mathrm{c}} \\
232^{\mathrm{d}}\end{array}$ & $\begin{array}{l}77.416 \\
84^{\mathrm{c}}\end{array}$ & $\begin{array}{l}76.242 \\
93^{\mathrm{c}} \\
77.4^{\mathrm{d}}\end{array}$ & $\begin{array}{l}141.56 \\
142^{\mathrm{c}} \\
129^{\mathrm{d}}\end{array}$ & 5.848 & $\begin{array}{l}210.876 \\
224^{\mathrm{c}}\end{array}$ & $\begin{array}{l}1.013 \\
0.95^{\mathrm{b}}\end{array}$ & 0.001 & $\begin{array}{l}1.690 \\
1.67^{\mathrm{d}}\end{array}$ & $\begin{array}{l}0.251^{\mathrm{c}} \\
0.24^{\mathrm{c}} \\
0.25^{\mathrm{d}}\end{array}$ \\
\hline
\end{tabular}

${ }^{\mathrm{a}} \operatorname{Ref}[6],{ }^{\mathrm{b}} \operatorname{Ref}[7],{ }^{\mathrm{c}} \operatorname{Ref}[8],{ }^{\mathrm{d}} \operatorname{Ref}[9]$
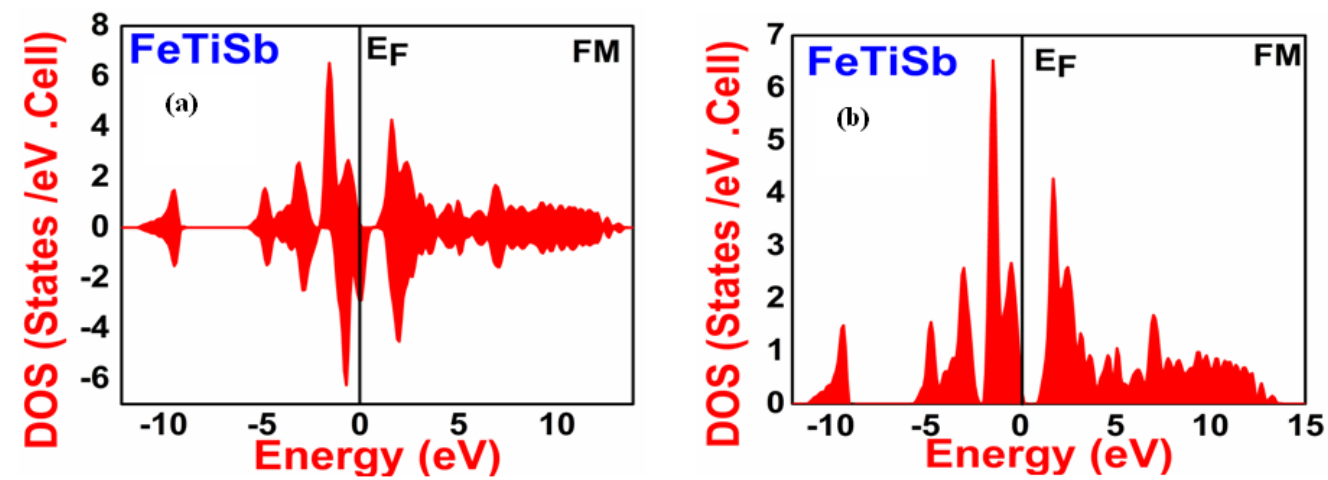

FIG .1. (a) \& (b). spin up ( $\uparrow$ and spin down $(\downarrow)$ density of states and Total density of states of FeTiSb

\subsection{Magnetic Properties}

Non-spin and spin polarized calculations are performed to check the magnetic stability of both FeTiSb and CoTiSb alloys at ambient pressure. Table 2 indicates that the FeTiSb is stable in ferromagnetic phase (FM) and CoTiSb are stable in the nonmagnetic (NM) phase, and their corresponding magnetic moments are found to be $0.906 \mu_{\mathrm{B}}$ and $0.001 \mu_{\mathrm{B}}$. The individual magnetic moment of Fe, Sb and Ti atoms are $1.195 \mu_{\mathrm{B}} 0.018 \mu_{\mathrm{B}}$ and $0.309 \mu_{\mathrm{B}}$. at normal pressure. Ti atom shows the negative magnetic moment whereas $\mathrm{Fe}$, sb atoms give the positive magnetic moment. The reduction of total magnetic moment of FeTiSb alloy is accompanied by the hybridization between the negative spin moments of Ti and the Fe and $\mathrm{Sb}$ atoms.

\section{Conclusion}

In conclusion, first principles calculations have been performed using Vienna ab-initio simulation package with GGA-PBE to investigate the structural, electronic and mechanical properties of titanium based magnetic materials with two possible phases. FeTiSb is stable in ferromagnetic phase (FM) and CoTiSb is stable in the nonmagnetic (NM) phase phase. The calculated mechanical properties of FeTiSb and CoTiSb alloys say that the CoTiSb is the stiffer material than FeTiSb. The electronic structure reveals that FeTiSb having half metallic ferromagnets material at normal pressure. In CoTiSb alloy is a semiconductor. The magnetic moment of both FeTiSb and CoTiSb alloys are also estimated.
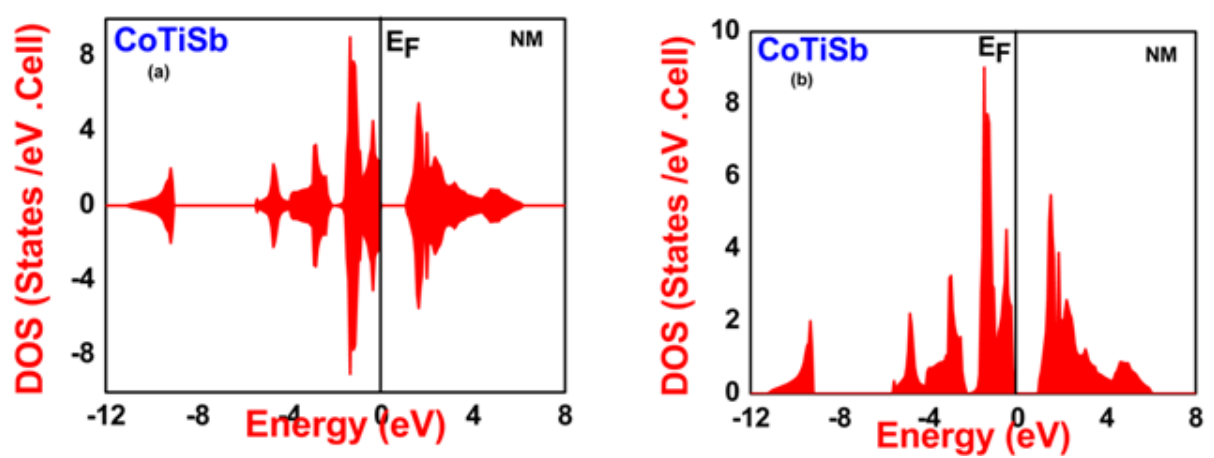

FIG .2.(a) \& (b) spin up ( $\uparrow$ ) and spin down $(\downarrow)$ density of states and Total density of states of CoTiSb

\section{Acknowledgements}

National Conference on Current Advancements in Physics $3^{\text {rd }} \& 4^{\text {th }}$ February 2017

Department of Physics, St. John's College, Palayamkottai-627 002, Tamilnadu, India. DOI 10.9790/4861-17002023437 
We thank our college management for their constant encouragement.

\section{References}

[1]. Mitsuo Niinomi, Science and Technology of Advanced Materials 4, 2003, 445-454.

[2]. Kathy Wang, The use of titanium for medical applications in the USA, Materials Science and Engineering, A, 213, 1996, 134- 137.

[3]. John P.Perdew, Kieron Burke and Matthias Ernzerhof, Generalized Gradient Approximation Made Simple, Physical Review Letters 77 (18), 1996, 3865-3868.

[4]. $\quad$ G. Kresse and J.Furthmuller, Physical ReviewB 54 (16), 1996, 11169-11186.

[5]. Bin Xu, Jing Zhang, Jianchu Liang, Guoying Gao and Lin Yi, Journal of Solid State Chemistry, 192, 2012, 351-355.

[6]. M.Ibrir, S.Lakel, S.Berri, S.Alleg and R.Bensalem, Ab Initio study of structural, electronic, magnetic alloys: XTiSb ( $\mathrm{X}=\mathrm{Co}, \mathrm{Ni}$ and $\mathrm{Fe})$, Proc, $4^{\text {th }}$ Inter.Conf in Advances in Applied physics and Materials Science (APMAS), 2014, 020046-1- 020046-7.

[7]. Janusz Tobola, Jacques Pierre, Electronic phase diagram of the XTZ (X= Fe, Co, Ni ; T=Ti, V, Zr, Nb, Mn ; Z=Sn, Sb) semi -Heusler compounds, Journal of Alloys and Compounds 296, 2000, 243-252.

[8]. Siham Ouardi, Gerhard H. Fecher and Claudia Felser, Electronic structure and optical, mechanical and transport properties of the pure, electron- doped and hole- doped Heusler compound CoTiSb, Physical Review B 86, 2012, 045116-1-045116-13.

[9]. G.Rogl, A.Grytsiv, M.Gurth, A.Tavassli, C. Ebner, A.Wunschek, S.Puchegger .Soprunyuk, W.Schranz, E.Bauer, H.Muller, M.Zehetbauer, Mechanical properties of half - Heusler alloys, Acta Materialia 107, 2016, 178-195. 